\title{
Using Systematic Review and Evidence Banking to Increase Uptake and Use of Aquatic Science in Decision-Making
}

Micah G. Bennett ${ }^{1}{ }^{*}$, Sylvia S. Lee ${ }^{1}$, Kate. A. Schofield ${ }^{1}$, Caroline Ridley $^{1}$, Susan B. Norton ${ }^{1}$, J. Angus Webb ${ }^{2,3}$, Susan J. Nichols ${ }^{3,4}$, Ralph Ogden ${ }^{4}$, Alexandra Collins ${ }^{5}$

1. National Center for Environmental Assessment, U.S. Environmental Protection Agency, Office of Research and Development, Washington, D.C. 20460

2. Department of Infrastructure Engineering, University of Melbourne, Parkville, Victoria 2010, Australia

3. Centre for Evidence Informed Policy and Practice, Australia (https://ceipp.org.au/)

4. Institute for Applied Ecology, University of Canberra, Bruce, Australian Capital Territory, 2617

5. Center for Environmental Policy, Imperial College, London, SW71NA, UK

*Author to whom correspondence should be addressed: bennett.micah@epa.gov

\begin{abstract}
To support sound decision-making in environmental management, we need rigorous, defensible, and transparent synthesis of scientific evidence. The Association for the Sciences of Limnology and Oceanography (ASLO) and associated aquatic science societies are leaders in applying science to decision-making, and yet many environmental decisions are still at risk of having to be made without a comprehensive, well-synthesized evidence base to support them. In this article, we discuss two synergistic approaches that can help science inform decisionmaking: systematic review and evidence banking. Our aim is to promote the use of these approaches, and to enlist support and action from you, the aquatic science community. We propose that you can improve the use and uptake of science in decision-making by making your research more compatible with synthesis efforts by: considering risk of bias when designing your study and reporting results; reporting all relevant contextual information; analyzing your data using standard effect size approaches; and publishing your raw data. Awareness of how primary research feeds into informing policies can help you broaden the impact of your research, making it more directly relevant to decision-making and more likely to contribute to the protection of aquatic ecosystems.
\end{abstract}

\section{Introduction}

This is the author manuscript accepted for publication and has undergone full peer review but has not been through the copyediting, typesetting, pagination and proofreading process, which may lead to differences between this version and the Version of Record. Please cite this article as doi: $10.1002 /$ lob.10283

This article is protected by copyright. All rights reserved. 
As aquatic scientists, we increasingly realize the need for sound science to inform decisionmaking so that sound science can contribute to effective management and protection of aquatic ecosystems. ASLO highlighted this need by bringing together a set of articles in a special online 'Science Meets Policy' collection that highlights decision-relevant papers from recent issues of Limnology and Oceanography Bulletin. Scientific societies like ASLO are increasing efforts to promote the use of science in the decision-making process, for example by forming policy committees, making public statements on scientific issues, responding to public comment requests for policy documents, holding Congressional briefings, publicizing papers with policy-relevant findings, and creating summaries of decision-relevant science topics for non-technical audiences. In 2010, several aquatic science societies formed the Consortium of Aquatic Science Societies (CASS) to coordinate scientifically valid and timely responses to science-policy issues that represent the views of aquatic scientists. There are more than 20,000 scientists represented by CASS, and ASLO is among the ten societies that make up the organization (https://aquaticsocieties.org/).

Despite these efforts, the application of science to environmental decision-making remains a challenging process (Head 2017). One of the challenges that environmental decision-makers face, at local to national scales, is information overload. Recent estimates suggest that 2.5 million scientific articles are published each year, representing an increase of $\sim 3 \%$ each year for the last two centuries (Ware and Mabe 2015). As scientific publication rates skyrocket, overwhelmed decision-makers, who are often not scientists, may not believe that a comprehensive, well-synthesized evidence base that addresses their issue is achievable. This is particularly important given that special interest groups are willing to step in and offer advice that may align with their values, but not necessarily all the available science.

To support sound decision-making in environmental management, we need rigorous, defensible, and transparent synthesis of scientific evidence. In this article, we discuss two synergistic approaches that are increasingly being used to help science inform decision-making: systematic review and evidence banking. Our aim is to promote the use of these approaches, and to enlist support and action from the aquatic science community. We explain what systematic review and evidence banking entail, as well as the challenges we have encountered while applying these approaches to environmental management. We present potential solutions to these challenges, some of which ask scientists to rethink or modify how they conduct and communicate research (Fig. 1). Even if you are not a scientist with direct involvement in policy discussions, this information can broaden the impact of your research, making it more directly relevant to decision-making and more likely to contribute to the protection of aquatic ecosystems. 


\section{Systematic Review}

As students, we start with a thorough review of the literature to understand a topic. We might even attempt to conduct what we would describe as a "systematic" review of the literature by reading and citing as many papers as possible. However, systematic review is much more than the large literature review common in dissertations and journals. Systematic review (SR; Fig. 2) is a structured and highly documented process for gathering and synthesizing evidence from existing studies to form conclusions (Higgins and Green 2011). The highly structured nature of the approach ensures completeness and reduces bias; the high level of documentation lends transparency and repeatability to the process. Systematic review is also different from reviews conducted by the National Academy of Sciences, which rely on carefully chosen experts to provide objective and independent information but do not require that the information comes from a comprehensive literature search (see

http://www.nationalacademies.org/studyprocess/index.html). Even if you are not interested in conducting a systematic review, it is useful to know what it is and why it is used to synthesize science to support policies. The lessons we learned from conducting a series of systematic reviews can help you increase the likelihood that your primary research is included in such efforts (Fig. 1).

Systematic review grew out of the push for evidence-based medicine in the late 1970 s and early 1980s and initially focused on evaluating effectiveness of treatments in clinical trials. Although SR is still predominantly applied to assess the effectiveness of medical interventions, it is increasingly being used to explore causal relationships, incidence of disease or condition, and other questions. Its use has broadened to include more disciplines, such as sociology, public health, education, economics, and, more recently, environmental management and conservation. Examples of environmental SRs published in the journal Environmental Evidence include the effects of cropland conversion, phasing out chemical production, or restoring wetlands (see http://www.environmentalevidence.org/completed-reviews).

Systematic review is especially useful when there are inconsistent findings in the literature or when an action or decision will be highly visible, controversial, or potentially subject to legal challenge. Systematic review allows decision makers to have a synthesis of relevant information at their fingertips, so they can make definitive statements about issues and have confidence that decisions reflect a transparent and comprehensive body of scientific evidence. Systematic review findings are also useful for researchers and funding agencies in identifying knowledge gaps (and thus potentially fruitful areas of research) and uncovering potential methodological 
improvements in a given field of study. At times, depending on the nature and volume of studies on a subject, SRs may be unable to provide certainty about the effectiveness of an intervention or the strength of a relationship. However, even when this is the case, SRs can provide valuable information by summarizing variability and uncertainty among studies.

Systematic review, as with other types of assessments, begins with scoping and problem formulation (Fig.2). During scoping, the SR team considers needs of the decision-makers to specify review parameters (e.g., the timeline). Problem formulation results in a targeted question that addresses the specific factors to be evaluated. Not all types of questions are suitable for SR (Cook et al. 2017). A broad question (e.g., What are the effects of nutrients on river ecosystems?) will return voluminous and diffuse research results that are time-consuming to screen and difficult to synthesize. Broad questions should be iteratively refined with decision-makers to target specific parameters of interest, potentially resulting in multiple, discrete SRs. Co-developing review questions with decision-makers also increases the salience, credibility, and legitimacy of the information produced, and in turn improves the efficiency of knowledge transfer (Cash et al. 2003; Collins et al. 2015). A systematic map of the available literature may be a good first step to refine initially broad questions (CEE 2018). A systematic map (Fig. 2) is an evidence synthesis method that summarizes the number of studies in prespecified 'bins' of interest (see "Challenges and Opportunities..." section below).

Next, the SR team develops a detailed protocol a priori, which outlines how they will conduct the remaining SR steps. In the protocol, the SR team identifies the literature sources they will search, documents keyword search strings, and describes processes for (1) screening papers for relevance, (2) extracting data, (3) assessing study quality, and (4) checking for consistency among team members at each of the above steps. Best practice across fields is to publish this protocol prior to conducting the review, to minimize the potential for bias (Higgins and Green 2011). Many organizations, journals, or other publication outlets (e.g., bioRxiv) now publish or register SR protocols, although few are targeted at ecological sciences (but see Environmental Evidence).

The real work begins after completing the protocol. The SR team implements the literature search strategy outlined in their protocol (Fig. 1). The search should include articles from academic databases and gray literature sources (e.g., agency reports, government websites), as well as other approaches such as requests to colleagues, social media, or listservs. This is a departure from most traditional literature reviews in ecology, but ensures a comprehensive body of literature free (as much as possible) from publication bias, the tendency for only studies with significant results to be published in journals. 
The SR team then screens the references returned from the searches for relevance and notes why any studies are excluded using criteria in the protocol (e.g., an irrelevant response or endpoint). Relevant studies move into data extraction, where the SR team identifies and records key information from each study (e.g., effect sizes, geographic and contextual variables). In SR, individual studies also undergo critical appraisal or quality assessment (Haddaway et al. 2015) focusing on aspects of random and systematic error (bias). This contrasts with most traditional reviews, which treat each study as equal (or weight studies by their variance in meta-analysis). Although critical appraisal frameworks are numerous and welldeveloped in medical fields, currently most published SRs in ecological fields develop their own. However, recently some general frameworks have appeared that provide a good starting point for considering study quality (e.g., Mupepele et al. 2016).

After completing the above stages, the focus turns to synthesis. The SR team conducts data analysis, often using meta-analysis when data are mostly quantitative. Then, they evaluate critical appraisal information to determine confidence in the evidence and determine if findings are sensitive to study quality, environmental modifiers, or other features of the evidence base (sensitivity analysis). Finally, the SR team considers all the information to develop conclusions about the focal question.

In environmental management and ecology, the primary international organization advancing SR methods is the Collaboration for Environmental Evidence (CEE;

www.environmentalevidence.org). A not-for-profit community of practice, CEE promotes SR by organizing conferences, working groups, and independent evidence centers. Perhaps its most important functions are the development of SR guidelines and publication of an open-access journal of SRs and systematic maps, protocols, and articles related to SR methodology and practice.

\section{Evidence Banking}

Systematic review relies on comprehensive searches of a large literature base, and often generates a substantial volume of information and data from relevant studies. The SR team extracts key data from relevant studies, including contextual information (e.g., country, climate) and results (e.g., effect sizes). Although the SR team extracts a body of evidence pertinent to a specific question, much of the extracted information could be used to answer other questions.

Ready access to an evidence 'bank' could increase the use of SRs in environmental decisionmaking. Data extraction is typically the most resource-intensive part of SR, and the time required to conduct a SR is often cited as the biggest impediment to the uptake of the SR 
approach (based on informal feedback). In three SRs currently in progress by some of the authors of this paper (see Case Study below), it has taken 130 minutes per article, on average, to extract $>40$ environmental, study design, and other context variables of interest. Banking these pieces of extracted evidence for use in future studies can help reduce the costs of SRs (in terms of time and/or money). Given the resource investment that SRs represent, it makes sense to have a central repository, or evidence bank, of individual studies and extracted information, rather than having each new review or meta-analysis duplicate data extraction already completed by others.

Currently there are several public database options for storing raw data from individual studies (e.g., Dryad), and this practice is gaining acceptance in ecological publishing. Although organizations like CEE require publication of screening results and extracted data from SRs in appendices, there are few options for storing the detailed information in centralized repositories, as opposed to individual supplemental files, to make it easily accessible and useable.

\section{Challenges and Opportunities for Greater Use of SRs and Evidence Banking in Aquatic Sciences}

Many aspects of SR and evidence banking, especially when applied to environmental science and management, contribute to the most common complaint about the methods: the time investment required. Decision-makers often want information over broad questions or topics to get as much evidence as possible, leading to large bodies of literature that need screening, data extraction, and synthesis. Environmental questions often can involve numerous potential endpoints, given that stressors typically affect multiple levels of biological organization and that researchers use many methods to measure effects at each level. Ecological systems, and many of the typical study designs employed to investigate them, involve many modifying and confounding factors that can increase the amount of data that needs to be extracted from each study (e.g., many contextual environmental variables) and complicate evaluation of study quality. Study quality evaluation itself can be time consuming and is not a widespread practice in traditional ecological literature reviews. The diversity of complicated designs used in ecological research, and the higher frequency of observational versus experimental studies, further complicate this process, since these designs are not often considered explicitly in established study quality approaches from medicine. Further, even more rapid methods that have been developed to at least partially address the time investment challenge typically require months to complete (Collins et al., 2015). 
If SR is the 'gold standard' for determining what we know about a specific scientific or management question and then applying this knowledge to decision-making, the factors contributing to the 'time challenge' must be addressed. Decision-making and policy windows in environmental management are often short. If a SR team cannot deliver the 'gold standard' in a timely manner, their hard work could be wasted (Rose et al. 2017). Below, we highlight some ways of addressing the time challenge.

Carefully scope the question. The more precisely defined the systems, stressors, and outcomes of interest, the more specific and efficient the searching and screening process will be. When possible, co-develop the question with end users to ensure that it meets decision makers' needs.

Limit full data extraction to studies meeting minimum quality standards. If study quality is evaluated first, and some minimum acceptable quality parameters are established a priori, this will focus one of the most time intensive steps (extraction) on the smaller subset of studies most likely to be useful for synthesis. However, this will limit the ability to determine if inclusion of studies without such minimum quality characteristics affects review conclusions and will decrease sample sizes for meta-analysis.

Use technological innovations for screening and data extraction (Westgate et al. 2018, Kohl et al. 2018). Machine learning and automation techniques are increasingly being used in SR, especially at the screening stage, with several software packages employing these approaches (e.g., SWIFT-Active Screener, DistillerSR). Training a machine-learning algorithm with a subset of include/exclude decisions allows the algorithm to prioritize relevant papers and estimate when all relevant studies have been found. Automated data extraction is currently less developed as an off-the-shelf functionality, but is currently implemented in a few software packages (e.g., colandr).

Consider if other review methods are better fit for the purpose of the assessment. Review methods that provide results more rapidly than SR may be more appropriate, or more 'fit-forpurpose,' for the question of interest (Cook et al. 2017). Systematic mapping is a broad evidence review describing a few details about each study and showing 'hotspots' of literature based on an intervention/stressor across outcomes of interest (McKinnon et al. 2015, Haddaway et al. 2016). Because they require extraction of only a limited amount of information from each study and do not typically evaluate quality, systematic maps can be completed more quickly and can serve as a scoping tool for more targeted SRs. Other rapid methodologies seek to limit the number of sources searched, the amount of information extracted, and the aspects of quality evaluated to more rapidly assess a body of evidence on a topic, given the needs of 
the decision maker (Dicks et al. 2017, Webb et al. 2017). For instance, a Quick Scoping Review does not evaluate study quality and limits data extraction, while a Rapid Evidence Assessment focuses on a limited number of quality aspects and searches fewer resources (Collins et al. 2015). Rapid methods are under-developed in ecological disciplines but are of increasing interest to practitioners and decision makers. Any type of literature-based evidence review can also be made more reliable by applying some of the methodological rigor of SR, such as clear and thorough documentation of the processes followed at each step (Haddaway et al. 2015).

Promote and use evidence banking databases. Over the long term, promoting evidence banking efforts in ecological disciplines will speed the process of synthesizing literature-based evidence (Ziegler et al. 2015). Although quality assurance efforts will always be necessary, and additional information from papers may be desired in addition to what is currently captured in an evidence bank, substantial time savings will result when basic contextual, design and results information are already available for relevant studies.

SR teams need not be the only participants in evidence banking, nor are they the most efficient evidence extractors. Allowing individual researchers to enter results of their own studies into an evidence bank could potentially save substantial time and reduce inaccuracies, since study authors are most familiar with their own work. To make our science useful for decision-making, we should be doing everything we can to support accurate and timely synthesis of our collective knowledge. If more researchers contribute to evidence banking, we can make more accurate information available to speed up the SR and synthesis process.

A recent effort, piloted in the journal Freshwater Science, is notable in that it integrates this metadata banking process into the publication process to allow authors to enter data about their own studies while also allowing any user to enter information from a paper - including results of SRs or other synthesis efforts (Norton et al. 2018). The EcoEvidEx (Ecological Evidence Exchange) initiative takes a standardized set of data, entered by authors during the postacceptance phase of the manuscript publishing process at Freshwater Science or by other users at any time. These data are then accessible for existing, public evidence repositories (e.g., Eco Evidence, USEPA's CADLink). This information can be downloaded by other users for synthesis efforts, increasing accessibility of research findings and speeding up the process of applying results to decisions. This system could easily be extended to other journals in the aquatic sciences. EcoEvidEx is currently in a pilot phase, and anyone can enter information through links from the Society for Freshwater Science webpage.

Making YOUR Science Accessible for Decision-Making 
Scientific practice is different from environmental management, and not every researcher is interested in or has time for conducting evidence synthesis in a decision-making context. However, the following suggestions on how to make primary research results more useful for synthesis, based on our experience with SR and other synthesis efforts, may make the process less onerous (see additional considerations in Cooke et al. 2017).

Case study. We start with an example from a series of ongoing SRs to illustrate several points. The primary focus of the reviews is the effects of total nitrogen (TN) and total phosphorus (TP) (stressors) on multiple biological endpoints (responses) in streams and rivers (see Bennett et al. 2017 for the chlorophyll- $a$ endpoint protocol). We targeted TN and TP in part to limit the volume of literature to what was deemed practical, and in part because these are the primary nutrient components for which numeric nutrient criteria are being derived in the United States. While searching and screening the literature returned by our searches, we realized that most stream ecology experiments focused on readily-cycled nutrient forms and did not measure (or did not report) TN or TP concentrations. Out of 100 studies from a separate Web of Science search for experimental nutrient addition studies in mesocosm and field settings (and bibliographies of returned articles), only $3 \%$ and $5 \%$ of studies reported TN and TP, respectively. Since well-designed experimental studies are generally considered of higher quality than observational studies due to their ability to control for confounding environmental variables, the literature base for our synthesis question automatically began with studies with greater risk of confounding and bias (i.e., more observational than experimental studies). This is of course a result of the narrower scope of our review; however, given that states are using TN and TP to develop nutrient criteria, the lack of data on these nutrient measures in most experimental studies leads to some difficulty in combining and comparing with a synthesis of TN and TP effects. This brief example suggests some important considerations for making primary research results more useful in a synthesis context.

Report or publish all relevant contextual information. Several experimental studies in the above example reported that TN or TP were measured but did not provide details on concentrations in treatment/control units or other important information. Simply reporting mean TN or TP concentration for control and treatment units could have provided enough information to characterize relationships in terms of these key variables (e.g., \% dissolved of total). In our SRs, many studies reported collecting a variety of other environmental measurements that were not reported in the results. Contextual information that may not be the focus of the paper can provide critical information on factors that modify ecological relationships across a body of literature. Data that are collected in association with a field sampling campaign or experiment, even if they are not analyzed, can be uploaded as supplementary material or to one of several raw data repositories. At minimum, if a variable is 
important enough to be mentioned in the methods, authors should consider including raw data or summary statistics as supplementary material.

Analyze data using standard effect size approaches when appropriate, and publish raw data when not. Most ecological and environmental management research relies on quantitative analysis of data related to the question of interest. However, given the diversity of analysis techniques available, there is a large amount of heterogeneity in how data are analyzed and how results are reported. For synthesis questions answerable using quantitative meta-analysis, this heterogeneity can pose a significant challenge. Although there are many forms of effect size - the primary data point from a study used in a meta-analysis - all effect sizes must generally have known distributions, and synthesis is easier when results are analyzed using a fairly restricted set of approaches. Researchers should certainly not limit themselves only to these more standardized effect sizes; however, they could consider reporting statistics of simpler tests (such as correlation or simple linear regression), or including raw data for calculation of such statistics, to make quantitative synthesis easier. For instance, a large body of literature found during the case study SRs examined biotic assemblages and environmental covariates using ordination approaches (e.g., non-metric multidimensional scaling). Although these studies show links between nutrients and stream biota, it is difficult to design a quantitative synthesis of these results with more traditional stressor-response type studies using regression, correlation and the like without in-depth statistical work. By providing complementary analyses or raw data, authors of these kinds of studies could aid translation of results to answer a broader synthesis question.

Consider 'risk of bias' when designing studies and reporting results. Systematic reviews evaluate the "quality" of individual studies to determine the effects of that quality on findings. "Quality" is a general term that can include elements of internal validity (soundness of the research methods) and external validity (relevance of research to particular context or question). Most quality evaluations focus specifically on internal validity and the "risk of bias" the potential for features of the study to contribute to systematic bias in its results - in several domains. Researchers can take many steps during study design to reduce risk of bias. One simple action that can improve quality evaluation is full and accurate reporting of methods and results. By far the most common case we have seen in study quality evaluation is studies that do not provide enough details to evaluate particular areas of bias. A study may not detail how sites were selected, or provide details on sampling (e.g., methods, frequency, statistical treatment, sample size in analysis), or provide information other details that are critical for study appraisal. One particular bias domain - reporting bias - focuses on the potential that a study selectively reports results. This is another common situation, where a study suggests that multiple statistical tests were conducted in the methods but reports only a small subset (e.g., 
only statistically significant results). These bias issues are easily addressed by including supplementary material of all results, which can improve the ability to evaluate reporting and other quality areas.

\section{Conclusion}

At a time when environmental decision-making is increasingly complex and publicly scrutinized, science needs a strong, defensible, and transparent voice in the discussion. Systematic review, evidence banking, and other synthesis efforts, if designed to fit decision needs and timelines, can aid translation and application of scientific research to decision-making. Lessons learned from SR and evidence banking can also be applied to the design and communication of individual research efforts, giving them more impact and transparency. Decisions often are required when there is insufficient time to undertake further research and potentially without access to the best-available evidence. Such situations increase the probability of adopting potentially inappropriate management options. Methods that facilitate the use of the bestavailable evidence could inform a compelling scientific argument that reduces inferential uncertainty, clarifies gaps and uncertainties in understanding, and strengthens confidence in decision-making.

\section{Acknowledgments}

We thank April Luke (USEPA) for reviewing a previous draft of the manuscript and are grateful to the Editors for their interest and support during writing and publication. DISCLAIMER: The views expressed in this paper are those of the authors and do not necessarily reflect the views or policies of the US Environmental Protection Agency. Mention of trade names or commercial products does not constitute endorsement or recommendation for use.

\section{References}

Cash, D.W., W.C. Clark, F. Alcock, N.M. Dickson, N. Eckley, D.H. Guston, J. Jager, and R.B. Mitchell. 2003. Knowledge systems for sustainable development. Proceedings of the National Academy of Sciences 100: 8086-8091.

CEE (Collaboration for Environmental Evidence). 2018. Guidelines and standards for evidence synthesis in environmental management. Version 5.0. Collaboration for Environmental Evidence, Bangor, Wales. Available at www.environmentalevidence.org/information-forauthors. 
Collins, A.M., D. Coughlin, J. Miller, S. Kirk. 2015. The Production of Quick Scoping Reviews and Rapid Evidence Assessments: A How to Guide. Joint Water Evidence Group, Department of Environment and Rural Affairs, United Kingdom. Available at:

https://assets.publishing.service.gov.uk/government/uploads/system/uploads/attachment dat a/file/560521/Production of quick scoping reviews and rapid evidence assessments.pdf

Cook, C.N., S.J. Nichols, J.A. Webb, R.A. Fuller, R.M. Richards. 2017. Simplifying the selection of evidence synthesis methods to inform environmental decisions: A guide for decision makers and scientists. Biological Conservation 213: 135-145.

Cooke, S.J., K. Birnie-Gauvin, R.J. Lennox, J.J. Taylor, T. Rytwinski, J.L. Rummer, C.E. Franklin, J.R. Bennett, and N.R. Haddaway. 2017. How experimental biology and ecology can support evidence-based decision-making in conservation: avoiding pitfalls and enabling application. Conservation Physiology 5: 1-14. doi 10.1093/conphys/cox043.

Dicks L.V., N. Haddaway, M. Hernández-Morcillo, B. Mattsson, N. Randall, P. Failler, J. Ferretti, B. Livoreil, H. Saarikoski, L. Santamaria, R. Rodela, E. Velizarova, and H. Wittmer. 2017. Knowledge synthesis for environmental decisions: an evaluation of existing methods, and guidance for their selection, use and development - a report from the EKLIPSE project. Horizon 2020: European Union Funding for Research and Innovation. Available at: http://www.eklipsemechanism.eu/apps/Eklipse data/website/EKLIPSE D3-1-Report FINAL WithCovers V6.pdf

Haddaway, N. R., C. Bernes, B.-G. Jonsson, and K. Hedlund. 2016. The benefits of systematic mapping to evidence-based environmental management. Ambio 45: 613-620.

Haddaway, N. R., P. Woodcock, B. Macura, and A. Collins. 2015. Making literature reviews more reliable through application of lessons from systematic reviews. Conservation Biology 29: 15961605.

Head. B.W. 2010. Reconsidering evidence-based policy: Key issues and challenges. Policy and Society 29: 77-94.

Higgins, J.P.T. and S. Green. 2011. Cochrane Handbook for Systematic Reviews of Interventions. Version 5.1.0. Updated March 2011. The Cochrane Collaboration. Available at: http://handbook-5-1.cochrane.org/

Kohl, C., E. J. McIntosh, S. Unger, N. R. Haddaway, S. Kecke, J. Schiemann, and R. Wilhelm. 2018. Online tools supporting the conduct and reporting of systematic reviews and systematic maps: A case study on CADIMA and review of existing tools. Environmental Evidence 7: 1-17. 
McKinnon, M. C., S. Cheng, R. Garside, Y. Masuda, and D. Miller. 2015. Map the evidence. Nature 528: 185-187.

Mupepele, A.-C., J. C. Walsh, W. J. Sutherland, and C. F. Dormann. 2015. An evidence assessment tool for ecosystem services and conservation studies. Ecological Applications, 26: 1295-1301.

Norton, S. B., J. A. Webb, K. A. Schofield, S. J. Nichols, R. Ogden, M. Bennett, S. Lee, C. Ridley, D. Gibbs, and A. Collins. 2018. Timely delivery of scientific knowledge for environmental management: a Freshwater Science initiative. Freshwater Science 37: 7-9.

Rose, D.C., N. Mukherjee, B.I. Simmons, E.R. Tew, R.J. Robertson, A.B.M. Vadrot, R. Doubleday, and W.J. Sutherland. 2017. Policy windows for the environment: tips for improving the uptake of scientific knowledge. Environmental Science and Policy, doi: 10.1016/i.envsci.2017.07.013.

Ware, M. and M. Mabe. 2015. The STM Report: An overview of scientific and scholarly journal publishing. STM: International Association of Scientific, Technical and Medical Publishers. Fourth Edition published March 2015; updated with minor revisions November 2015. Available at: https://www.stm-assoc.org/2015 0220 STM Report 2015.pdf

Webb, J. A., K. Scho, M. Peat, S. B. Norton, and S. J. Nichols. 2017. Weaving common threads in environmental causal assessment methods : toward an ideal method for rapid evidence synthesis Freshwater Science 36: 250-256

Westgate, M. J., N. R. Haddaway, S. H. Cheng, E. J. McIntosh, C. Marshall, and D. B. Lindenmayer. 2018. Software support for environmental evidence synthesis. Nature Ecology \& Evolution 2: 588-590.

Ziegler, C.R., J.A. Webb, S.B. Norton, A.S. Pullin, and A.H. Melcher. 2015. Digital repository of associations between environmental variables: A new resource to facilitate knowledge synthesis. Ecological Indicators 53: 61-69. 
Fig. 1.

\section{Ways to Make YOUR Science Accessible for Decision- Making}

- Understand how and why synthesis methods (e.g., systematic review) provide defensible scientific information for decisionmakers.

- Know how your primary research results can be included in synthesis efforts as "high quality evidence." You can:

o Report summary statistics or raw data of all measured environmental variables, to help place study results in context of potential modifying factors. These modifying factors may not be of primary interest to you, but they may be critical to others who want to apply your results.

o Consider including analyses with standard effect sizes compatible with meta-analysis methods, such as correlation coefficients and $R^{2}$.

o Report results of all statistical analyses you described in the methods section, even if they were not statistically significant.

o Publish raw data in publicly accessible repositories or as supplemental information for your article.

- Submit information about your study to an evidence bank. 
Fig. 2. An overview of the systematic review and systematic map processes and how they can use evidence from primary research to inform decision-making. Adapted from Boyles et al. 2016, OHAT/NTP/NIEHS.

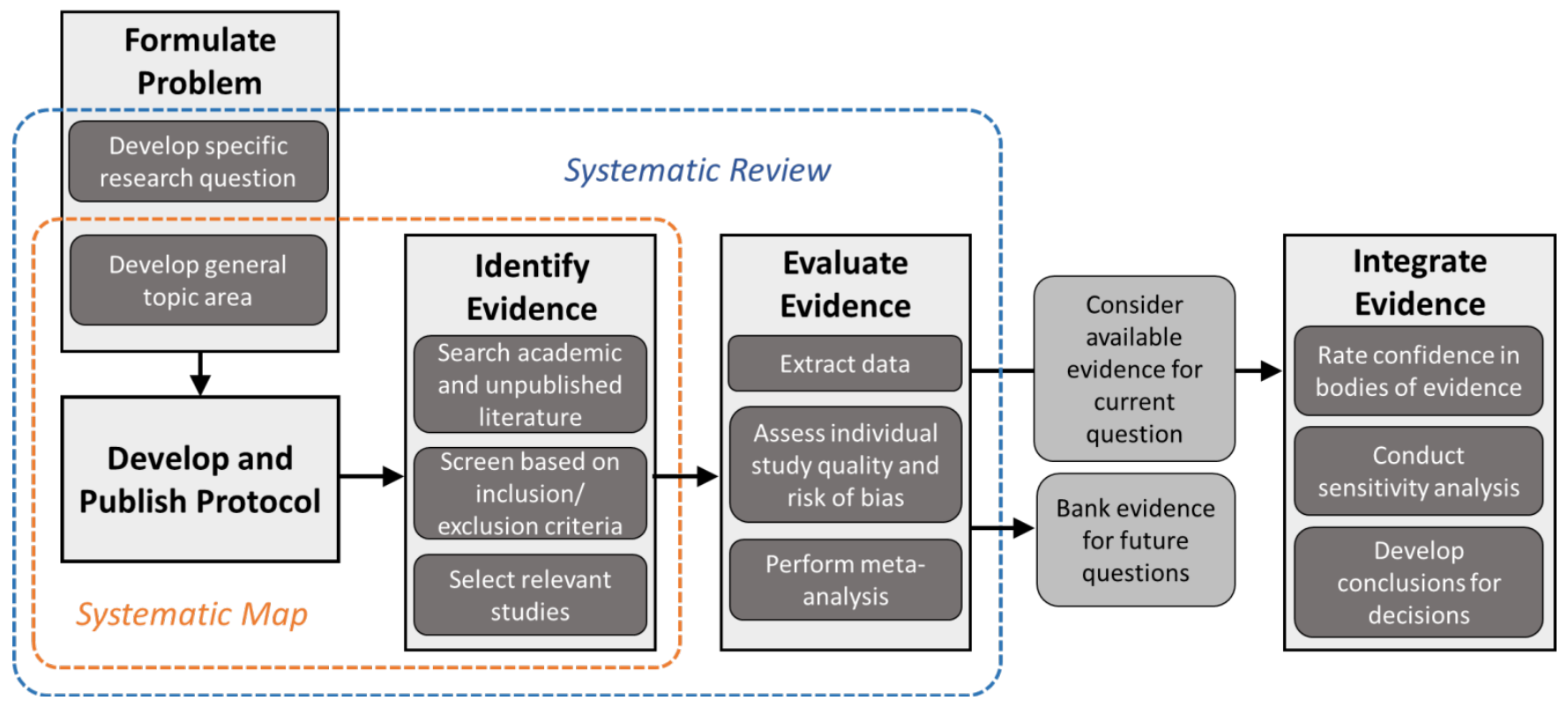




\section{University Library}

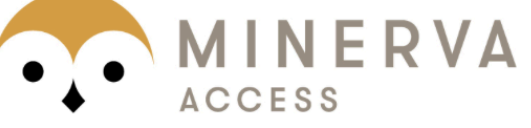

A gateway to Melbourne's research publications

Minerva Access is the Institutional Repository of The University of Melbourne

Author/s:

Bennett, MG;Lee, SS;Schofield, KA;Ridley, C;Norton, SB;Webb, JA;Nichols, SJ;Ogden,

R;Collins, A

Title:

Using Systematic Review and Evidence Banking to Increase Uptake and Use of Aquatic Science in Decision-Making

Date:

2018-11-01

Citation:

Bennett, M. G., Lee, S. S., Schofield, K. A., Ridley, C., Norton, S. B., Webb, J. A., Nichols, S. J., Ogden, R. \& Collins, A. (2018). Using Systematic Review and Evidence Banking to Increase Uptake and Use of Aquatic Science in Decision-Making. Limnology and Oceanography Bulletin, 27 (4), pp.103-109. https://doi.org/10.1002/lob. 10283.

Persistent Link:

http://hdl.handle.net/11343/284713 